\title{
Expression and role of PGP, BCRP, MRP1 and $M R P 3$ in multidrug resistance of canine mammary cancer cells
}

Karol M Pawłowski ${ }^{1,2}$, Joanna Mucha ${ }^{1}$, Kinga Majchrzak ${ }^{3}$, Tomasz Motyl ${ }^{1}$ and Magdalena Król ${ }^{*}$

\begin{abstract}
Background: In both women and female dogs, the most prevalent type of malignant neoplasm is the spontaneous mammary tumor. In dogs, half of these are malignant. The treatment of choice for the canine patients is surgical mastectomy. Unfortunately, it often fails in high-risk, locally invasive mammary tumors as of during the time of the surgery the micro-metastases are present. Moreover, there are neither large studies conducting to prove of the benefit from the chemotherapy in dogs nor established chemotherapy treatment protocols available. Additionally, the effectiveness of each individual chemotherapeutic agent and drug resistance of canine mammary cancer have not yet been characterized. That has become the aim of our study, to assess the expression of PGP, BCRP, MRP1 and MRP3 in canine mammary cancer cell lines and to investigate their role in cancer resistance to vinblastine, cisplatin and cyclophosphamide with using RNAi approach.

Results: The results suggested that in canine mammary cancer, the vinblastine efflux was mediated by PGP and MRP1 proteins, cisplatin efflux was mediated by all four examined efflux pumps (PGP, BCRP, MRP1 and MRP3), whereas cyclophosphamide resistance was related to BCRP activity. RNAi silencing of these efflux pumps significantly decreased IC50 doses of the examined drugs in canine mammary carcinoma cells.
\end{abstract}

Conclusions: Our results have indicated the treatment of cells involving use of the siRNA targeting efflux pumps could be a beneficial approach in the future.

Keywords: Multidrug resistance, Chemotherapy, Canine mammary cancer, Vinblastine, Cisplatin, Cyclophosphamide, PGP, BCRP, MRP1, MRP3

\section{Background}

In both women and female dogs, the most prevalent type of malignant neoplasm is the spontaneous mammary tumor. In dogs, half of these are malignant. There is a threefold incidence in the canine, with middle aged, non-spayed female dogs being the most affected [1,2]. The early ovariectomy is thought to reduce the risk of mammary cancer development [3], however, the high morbidity and mortality rate due to ineffective treatment strategies makes this problem as being still actual. The treatment of choice for the canine patients is surgical mastectomy. Unfortunately, it is often recognized -

\footnotetext{
* Correspondence: magdalena_krol@sggw.pl

'Department of Physiological Sciences, Faculty of Veterinary Medicine, Warsaw University of Life Sciences - WULS, Nowoursynowska 159, 02-776, Warsaw, Poland

Full list of author information is available at the end of the article
}

unsatisfactory as in case of invasive cancer at the time of surgery the micro-metastases are present. Moreover, clinical studies reveal that after regional mastectomy $77 \%$ of dogs developed a new malignancy [4]. There are neither large studies conducting to prove to the benefit of chemotherapy in dogs nor established chemotherapy treatment protocols available. Moreover, the effectiveness of each individual chemotherapeutic agent and drug resistance of canine mammary cancer have not yet been characterized. The available data is conflicting, possibly due to the small number of studies performed, or the insufficient number of dogs used in these studies. Some veterinarians adapt human chemotherapy protocols; however these treatment modalities are often unsatisfactory due to similarities observed in recurrence time, time to metastasis and overall survival [5-7].

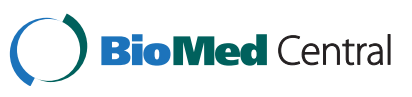


The resistance to a specific cytotoxic drug is ensured by the activity of efflux pumps that belong to the ABCtransporters super family. The most important in breast cancer are: PGP (P-glycoprotein), BCRP (Brest Cancer Resistance Protein), MRP1 (Multridrug resistance Protein 1) and MRP3 (Multidrug Resistance Protein 3). For example, in human cancer over-expression of PGP is linked to resistance to vinca alkaloids, anthracyclines, epipodophyllotoxins and also tubulin polymerizing drugs whereas MRP1 is responsible for resistance to vincristine and vinblastine, methothrexate, anthracyclines, etoposide, paclitaxel and irinotecan [8,9]. Unfortunately, expression of efflux pumps and their specific substrates have not been assessed in dogs yet. There is just one document published showing that BCRP can fully protect canine mammary cancer cells against doxorubicin [10].

That is why the aim of our study was to assess expression of PGP, BCRP, MRP1 and MRP3 in five canine mammary cancer cell lines and to investigate their role in cancer resistance to vinblastine, cisplatin and cyclophosphamide which are used in breast cancer treatment $[11,12]$. The examined drugs were selected based on the available data in the field of veterinary oncology. They presented positive results of cisplatin and cyclophosphamide treatment in canine mammary cancer $[5,7]$ whereas vinblastine was selected due to its general tolerance [13]. Moreover, these anticancer agents belong to various groups, thus we used them to observe efflux pumps activity during treatment with drugs from different groups.

We also treated canine mammary cancer cells with siRNAs specific for these efflux pumps to knock-down their expression. Treatment of cancer cells by using classical inhibitors of efflux pumps often fails [8,9], thus we used a novel approach to reduce their activity.

Our results have pointed out that treatment of patients with the supportive use of the siRNAs specific for efflux pumps may help with improving results of chemotherapy. Despite the useful function of RNAi therapeutics for disease treatment, it, yet - still requires the development of clinically suitable, safe and effective drug delivery vehicles, there are some promising data from ongoing clinical trials that give hope for their practical application in the future [14].

\section{Methods}

\section{Cell lines}

The cell lines used for the study have been previously used in other published research [14-18]. Two canine mammary adenocarcinoma cell lines (CMT-W1, CMT-W2), anaplastic cancer cell line (P114), simple carcinoma cell line (CMT-U27) and spindle-cell mammary tumor cell line (CMT-U309) had been examined. Cells were cultured under optimal conditions: in RPMI-1640 medium enriched with $10 \%(\mathrm{v} / \mathrm{v})$ heat-inactivated fetal bovine serum (FBS), penicillin-streptomycin (50 iU mL-1), and fungizone $(2.5 \mathrm{mg} \mathrm{mL}-1)$ (reagents obtained from Sigma Aldrich, USA), in an atmosphere of 5\% CO2 and 95\% humidified air at $37^{\circ} \mathrm{C}$.

\section{siRNA transfection}

The siRNA transfection procedure used in canine mammary cancer cells was described in details in our previously published studies [19-21]. The cell density, transfection reagent toxicity and transfection efficacy were optimized according to the procedure described in our previous manuscript: [19]. The canine (Canis lupus familiaris) pgp, bcrp, mrp1 and mrp3 sequences were obtained from Gene Bank with accession numbers: NM_001003215, DQ222459.1, NM_001002971, XM_54 8204.2, respectively. The siRNA duplexes were designed by http://www.sigmaaldrich.com/life-science/custom-olig os/sirna-oligos/sirna-design-service.html. The results were confirmed using two independent algorithms: Dharmacon (OligoWalk) and Ambion and two duplexes were used for further experiments (obtained from Sigma Aldrich) (Table 1). For each gene silencing the mixture of both duplexes was used $(30 \mathrm{pmol}+30 \mathrm{pmol})$. All the experiments with transfected cells were conducted 24-48 hrs after the transfection.

\section{Reverse-transcriptase qPCR}

Total RNA was isolated using a Total RNA kit (A\&A Biotechnology, Poland) according to the manufacturer's protocol. Isolated RNA samples were dissolved in RNase-free water. The quantity of isolated RNA was measured using NanoDrop (NanoDrop Technologies, USA). The mean concentration of RNA was $173 \mathrm{ng} / \mu \mathrm{l}$, and A260/280 ratio was between 1.8 and 2.0. The samples with adequate amounts of RNA were treated with DNaseI to eliminate DNA contamination. The samples were subsequently purified using RNeasy MiniElute Cleanup Kit (Qiagen). Finally RNA samples were analyzed on a BioAnalyzer (Agilent, USA) to measure final RNA quality and integrity. Only RNA with RIN (RNA Integrity Number) $>9$ was used for the further analyses. Primers used to detect the expression of pgp, bcrp, mrp1 and $m r p 3$ genes were designed using PRIMER3 software (free on-line access) and checked using Oligo Calculator (free on-line access) and Primer-Blast (NCBI database). The used sequences are listed in Table 2. rps19 and hprt genes were used as non-regulated references for the normalization of target gene expression [22,23]. Quantitative RT-PCR was performed using fluorogenic SYBR Green and the Sequence Detection System, Fast 7500 (Applied Biosystems). Data analysis was carried out using the 7500 Fast System SDS Software Version 
Table 1 Used siRNA sequences

\begin{tabular}{|c|c|c|c|}
\hline Gene & GenBank ID & First strand & Second strand \\
\hline \multirow[t]{2}{*}{$\overline{p g p}$} & NM_001003215 & CGAACUGUUGUUUCUUUGA[dT][dT] & UCAAAGAAACAACAGUUCG[dT][dT] \\
\hline & & CUUCCGAACUGUUGUUUCU[dT][dT] & AGAAACAACAGUUCGGAAG[dT][dT] \\
\hline \multirow[t]{2}{*}{ bcrp } & DQ222459.1 & GCCCAGGAGUCAAUGUAAC[dT][dT] & GUUACAUUGACUCCUGGGC[dT][dT] \\
\hline & & CGAAUAAUACCUGUAGCUA[dT][dT] & 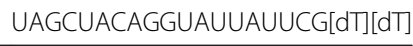 \\
\hline \multirow[t]{2}{*}{ mrpl } & NM_001002971 & GAAAGAGGCUCCCUGGCAA[d]][dT] & UUGCCAGGGAGCCUCUUUC[dT][dT] \\
\hline & & GGAGUAUUCAGAAACGGAG[dT][dT] & CUCCGUUUCUGAAUACUCC[d]][dT] \\
\hline \multirow[t]{2}{*}{ mrp3 } & XM_548204.2 & GGCUAUGACGGAGAGCCAA[dT][dT] & UUGGCUCUCCGUCAUAGCC[dT][dT] \\
\hline & & UGUCUACGCUGCCUUGGGA[dT][dT] & UCCCAAGGCAGCGUAGACA[dT][dT] \\
\hline
\end{tabular}

siRNA sequences used to pgp, bcrp, mrp1 and mrp3 genes silencing in canine mammary cancer cell lines. The canine (Canis lupus familiaris) pgp, bcrp, mrp1 and mrp3 sequences were obtained from Gene Bank with accession numbers: NM_001003215, DQ222459.1, NM_001002971, XM_548204.2, respectively. The siRNA duplexes were designed by http://www.sigmaaldrich.com/life-science/custom-oligos/sirna-oligos/sirna-design-service.html. The results were confirmed using two independent algorithms: Dharmacon (OligoWalk) and Ambion.

1.4.0.25 (Applied Biosystems, USA). The results were analyzed using comparative $\mathrm{Ct}$ method [24]. Relative transcript abundance of the gene equals $\Delta \mathrm{Ct}$ values $\left(\Delta \mathrm{Ct}=\mathrm{Ct}^{\text {reference }}-\mathrm{Ct}^{\text {target }}\right)$. Relative changes in transcript were calculated as $\Delta \Delta C t$ values $\left(\Delta \Delta C t=2^{-\Delta C t}\right)$. The experiment was conducted three times.

\section{Flow cytometry determination of rhodamine-123 accumulation}

Examined cancer cells $\left(5 \times 10^{6}\right.$ cells per ml) (control cells and cells treated with $p g p, b c r p, m r p 1$ and $m r p 3$-specific siRNAs) were incubated for $1 \mathrm{~h}$ at $37^{\circ} \mathrm{C}$ in the $1 \mathrm{mmol}$ of rhodamine-123 (obtained from Sigma Aldrich, Germany). After the incubation time, cells were washed twice, resuspended in ice cold PBS and kept at $4^{\circ} \mathrm{C}$ in the dark until analysis in the flow cytometer. At least 50000 cells per sample were counted and analyzed by flow cytometry (BD FACS Aria II, Becton Dickinson, USA). Cells shown in forward scatter and side scatter were gated and acquired through the fluorescence channel. The amount of fluorescence was plotted as a histogram within the gate. Data acquisition was performed using BD FACS Diva software (Becton Dickinson, USA) to determine mean fluorescence intensity values. This procedure has been published before [25]. Results represent the average of at least three independent experiments.

The overlay histogram was created using Flowing Software (Turku University, Finland), available at www. flowingsoftware.com.

\section{Cell viability assay (MTT-assay) and $\mathrm{IC}_{50}$ determination}

Cell viability (metabolic activity of viable cells) was quantified by MTT assay. Cells were seeded into 96-well plate (Nunc Inc., Denmark) at the density which ensured their 50-70\% confluence at the day of experiment and: 1) treated with vinblastine (at the following concentrations: 12.3, 36.9, 61.5, 123, 369, 615, 2 460, 4 920, $7380 \mathrm{nmol}$ ), cisplatin (at the following concentrations: 46.5, 83, 465, 830, 4 650, 8 300, 46 500, $83000166000 \mathrm{nmol}$ ) or cyclophosphamide (at the following concentrations: 425, 950, 1 900, 3 800, 19 000, $3800076000 \mathrm{nmol}$ ) (all drugs obtained from Sigma Aldrich, Germany), 2) transfected with $p g p$, bcrp, mrp1 and mrp3 -specific siRNA and then treated with the anticancer drugs as given above, 3)

Table 2 Primers used for RT-qPCR

\begin{tabular}{lllll}
\hline Gene symbol & Forward primer & Reverse primer & $\begin{array}{l}\text { Optimum annealing } \\
\text { temp. }\left({ }^{\circ} \mathbf{C}\right)\end{array}$ & $\begin{array}{l}\text { Optimum annealing } \\
\text { time (sec) }\end{array}$ \\
\hline pgp & GCTTAACACCCGGCTCACAGAC & TAAGAAAGCGGCACCAATAGAAAT & 72 & 10 \\
$b c r p$ & GAGCTCCTTGTGGTGAGAA & AGGTGATGGTCATGAGGAGA & 72 & 10 \\
mrp1 & TTACTTTCCCTCGTGCTG & TATTCAGGGACCAAAGGTCA & 72 & 45 \\
mrp3 & AGGATGGACCTGATGACAGA & AACTTGGGATCAGGAGACC & 72 & 30 \\
hprt & AGCTTGCTGGTGAAAGGAC & TTATAGTCAAGGGCATATCC & 59 & 6 \\
$r p s 19$ & CCTTCCTCAAAAATCTGGG & GTTCTCATCGTAGGGAGCAAG & 61 & 10 \\
\hline
\end{tabular}

Primer sequences used in this study and their annealing optimal temperature and time. The mRNA sequences of key genes were obtained from NCBI database. Primers were designed using PRIMER3 software (free on-line access) and checked using Oligo Calculator (free on-line access) and Primer-Blast (NCBI database). Primers sequences are listed in Table 1. hprt and rps19 genes were used as non-regulated reference genes for normalization of target gene expression [22,23]. 
treated with non-coding siRNA (scrambled control) and cyctotoxic drugs as given above. These concentrations of anticancer drugs were determined in course of our preliminary experiments (data not shown) and chosen as appropriate to determine IC50 doses in all of the examined cell lines.

Then, cells were incubated in $0.5 \mathrm{mg} / \mathrm{ml}$ tetrazolium salt MTT diluted in phenol red-free RPMI 1640 medium (Sigma Aldrich) for $4 \mathrm{hrs}$ at $37^{\circ} \mathrm{C}$. To complete solubilization of the formazan crystals, $100 \mu \mathrm{l}$ of DMSO (Dimethyl sulfoxide, Sigma Aldrich) was added to each well. Cells viability was quantified by measuring photometric absorbance at $570 \mathrm{~nm}$ in multi well plate reader Infinite 200 PRO Tecan ${ }^{\text {nu }}$ (TECAN, Mannedorf, Switzerland). All the samples were examined in triplicate, each experiment was conducted seven times $(n=21)$. Based on these results cytotoxicity was determined. It was expressed as a mean percentage decrease relative to unexposed control \pm S.D. Control values were set at $100 \%$ viability. Cytotoxicity data were fitted to a sigmoidal curve and a four parameter logistic model was used to calculate IC50, which is the concentration of agent which reduces cell growth by $50 \%$ under the experimental conditions (increasing apoptosis, necrosis or causing block in cell cycle). This analysis was performed using GraphPad Prism 5.0 (San Diego, USA). This method of IC50 analysis has been previously published [26].

\section{Apoptosis assay}

The Annexin V-FITC and propidium iodide (PI) dual staining was applied for apoptosis analysis. Control cells (1) and cells treated with (2) anticancer drug at IC50 dose and (3) transfected with pgp, bcrp, mrp1 and mrp3specific siRNA moreover treated with anticancer drug at IC50 dose were harvested by trypsinization. These cells, as well as the cells floating in medium (RPMI 1640 containing 10\% FBS) were stained with an Annexin V Kit (Becton Dickinson, USA) according to the manufacturer's protocol. The cells then were analyzed by flow cytometry (BD FACS Aria II, Becton Dickinson, USA) within $1 \mathrm{hr}$ after staining. Early apoptotic cells with exposed phosphatidylserine but intact cell membranes bound to Annexin V-FITC however excluded PI. Cells in late apoptotic stages were labeled with both Annexin V-FITC and PI, whereas necrotic cells were labeled with PI only. All samples were assayed in triplicate. The experiment was conducted at least twice.

\section{Statistical analysis}

The analysis for statistical purposes was conducted using Prism version 5.00 software (GraphPad Software, USA). The one-way ANOVA and Tukey HSD (Honestly Significant Difference) post-hoc test were applied as well as regression analysis. Determination of IC50 test has been used. The p-value $<0.05$ was recognized as significant, whereas, p-value $<0.01$ and p-value $<0.001$ as highly significant. The data was expressed as means $+/-$ S.D. For molarity calculations, the Molarity Calculator (GraphPad, USA) on-line platform was used.

\section{Results}

PGP, BCRP, MRP1 and MRP3 are expressed in canine mammary cancer cells and their expression level changes due to anticancer drug in vitro treatment

RT-qPCR analysis revealed that all of the examined cell lines express $p g p, b c r p, m r p 1$ and $m r p 3$ (Table 3). The pgp expression ranged between 3.50 in CMT-U27 cell line and 17.85 in CMT-W1 cell line. The bcrp expression was the lowest in CMT-U309 cell lines (11.86) whereas the highest in CMT-W2 cell line (36.00). The highest expression of mrp1 was observed in CMT-W2 cell line (24.02) whereas the lowest expression was noticed in CMT-U27 cell line (7.50). CMT-W2 cell line exposed the lowest expression of mrp3 (11.35). The highest expression of mrp3 was detected in CMT-U309 cell line (23.28).

Treatment of cancer cells using scrambled siRNA did not cause any significant effect on examined gene expression (Table 3), whereas treatment of these cells with pgp, bcrp, mrp1 or mrp3 -specific siRNAs caused significant decrease in transcript level of targeted genes (the mostly significant effect was noticed in case of $p g p$ and mrp1) (Table 3). The efficacy of silencing reaction was very high, as there was observed $88-99 \%$ decrease in pgp transcript level, $14-100 \%$ in bcrp, $94-99 \%$ in $m r p 1$ and $22-100 \%$ in $m r p 3$ expression (Table 3 ).

Interestingly, we observed that pgp expression increased significantly in all the examined cell lines after viblastine and cisplatin treatment at IC50 doses, whereas decreased after cyclophosphamide treatment at IC50 dose (Table 3). Vinblastine treatment increased mrp1 expression in all of the examined cell lines with exception of CMT-W2 cell line where its expression increased also after treatment with cyclophosphamide at IC50 dose (Table 3). In case of $b c r p$, we observed increased tendency in its expression after cyclophosphamide treatment and decreased in its expression after vinblastine treatment at IC50 dose (Table 3). We are as well able to point out the increase in mrp3 expression in all the examined cell lines after treatment with cisplatin at IC50 dose (Table 3).

Cancer cell treatment with pgp, bcrp, mrp1 and mrp3 specific siRNA decreases efflux pumps activity enhancing rhodamine- 123 accumulation

The rhodamine-123 efflux assay was conducted to confirm our results of gene expression silencing and their functional 
Table 3 Expression of pgp, bcrp, mrp1 and mrp3 in various experimental conditions

\begin{tabular}{|c|c|c|c|c|c|c|}
\hline \multirow{2}{*}{$\begin{array}{l}\text { Efflux } \\
\text { pump }\end{array}$} & \multirow[t]{2}{*}{ Sample } & \multicolumn{5}{|c|}{ Relative expression in cell lines } \\
\hline & & CMT-U27 & CMT-U309 & P114 & CMT-W1 & CMT-W2 \\
\hline \multirow[t]{6}{*}{ PGP } & ctrl & 3.50 & 9.20 & 5.00 & 17.85 & 16.42 \\
\hline & pgp siRNA & $0.45^{* * *}$ & $0.10^{* * *}$ & $0.50^{* * *}$ & $0.80^{* * *}$ & $0.16^{* * *}$ \\
\hline & non-coding siRNA & 3.44 & 9.10 & 5.10 & 7.750 & 16.31 \\
\hline & vinblastine & $9.50^{* * *}$ & $16.20^{* * *}$ & $14.70^{* * *}$ & $19.56^{* * *}$ & $21.52^{* * *}$ \\
\hline & cisplatin & $8.95^{* * *}$ & $35.20^{* * *}$ & $31.00^{* * *}$ & $36.08^{* * *}$ & $26.16^{* * *}$ \\
\hline & cyclophosphamide & $0.09^{* * *}$ & $2.30^{* *}$ & $0.22^{* * *}$ & $0.08^{* * *}$ & $2.87^{* * *}$ \\
\hline \multirow[t]{6}{*}{ BCRP } & ctrl & 19.84 & 11.86 & 23.52 & 23.70 & 36.00 \\
\hline & bcrp siRNA & $17.15^{* *}$ & $2.77^{* * *}$ & $2.77^{* * *}$ & $10.46^{* * *}$ & $0.00^{* * *}$ \\
\hline & non-coding siRNA & 19.00 & 11.20 & 22.90 & 23.00 & 36.18 \\
\hline & vinblastine & $14.84^{*}$ & $3.80^{* * *}$ & $11.80^{* * *}$ & $12.30^{* * *}$ & $13.24^{*}$ \\
\hline & cisplatin & $30.50^{* * *}$ & $22.16^{* * *}$ & $40.34^{* * *}$ & $36.57^{* * *}$ & $37.23^{*}$ \\
\hline & cyclophosphamide & $23.32^{* * *}$ & $33.53^{*}$ & $29.23^{* * *}$ & $24.50^{*}$ & $41.72^{* *}$ \\
\hline \multirow[t]{6}{*}{ MRP1 } & ctrl & 7.50 & 9.40 & 16.50 & 20.10 & 24.02 \\
\hline & mrp1 siRNA & $0.10^{* * *}$ & $0.60^{* * *}$ & $0.23^{* * *}$ & $0.20^{* * *}$ & $0.61^{* * *}$ \\
\hline & non-coding siRNA & 7.20 & 6.62 & 20.12 & 20.91 & 24.21 \\
\hline & vinblastine & $9.70^{* * *}$ & $7.00^{* * *}$ & $9.76^{* * *}$ & $35.01^{*}$ & $30.20^{* * *}$ \\
\hline & cisplatin & $15.70^{* * *}$ & $19.34^{* *}$ & $21.84^{*}$ & $30.61^{* * *}$ & $26.20^{* *}$ \\
\hline & cyclophosphamide & $2.50^{* *}$ & $0.09 * * *$ & $3.32^{* * *}$ & $\downarrow 0.40^{* * *}$ & $6.04^{* *}$ \\
\hline \multirow[t]{6}{*}{ MRP3 } & ctrl & 14.40 & 23.28 & 13.84 & 17.70 & 11.35 \\
\hline & mrp3 siRNA & $1.00^{* * *}$ & $0.00^{* * *}$ & $4.84^{* * *}$ & $13.90^{* *}$ & $7.15^{* *}$ \\
\hline & non-coding siRNA & 13.87 & 21.99 & 13.09 & 17.10 & 10.99 \\
\hline & vinblastine & 14.20 & 21.97 & 13.12 & 17.50 & 9.78 \\
\hline & cisplatin & $15.60^{*}$ & $32.20^{* * *}$ & $15.80^{*}$ & $21.30^{* * *}$ & $14.09^{*}$ \\
\hline & cyclophosphamide & 14.99 & 24.09 & 14.25 & 18.01 & 11.72 \\
\hline
\end{tabular}

Expression of efflux pumps in canine mammary cell lines in control conditions, after scrambled siRNA treatment and pgp, bcrp, mrp 1 and $m r p 3$-specific siRNA treatment as well as after treatment with vinblastine, cisplatin and cyclophosphamide at IC50 doses. p $<0.05$ was marked as*, p $<0.01$ was marked as **, and $\mathrm{p}<0.001$ was marked as ${ }^{* * *}$. One-way ANOVA followed by Tukey HSD post-hoc test were applied. Increase in expression was marked as red text, decreased in expression was marked as green text, whereas no change in transcript level was marked as black text.

inhibition. It showed that after knock-down of $p g p$, bcrp, mrp1 and mrp3 expression level, the rhodamine-;123 accumulation significantly increased and that shows decreased activity of the efflux pumps (Figure 1A, B). Treatment of examined cells with scrambled siRNA did not cause any significant effect (data not shown). We observed that siRNA treatment of CMT-U27 caused significant but slight effect on rhodamine-123 accumulation (mean fluorescence related to rhodamine-123 accumulation in control cells was 97, whereas in siRNA treated cells it was between 108 and 110). In other cell lines the effect was higher (Figure 1), for example in P114 cell line the mean fluorescence related to rhodamine-123 accumulation in control cells was only 87 (the lowest, comparing to other cell lines) however, after the treatment with $b c r p$-specific siRNA is was 128 (Figure 1). The highest effect of rhodamine-123 accumulation after siRNA treatment was observed in CMT-W2 cell line (148 after mrp3-specific siRNA treatment, 111 in control conditions).
Cancer cell treatment with siRNA specific to efflux pumps decreases drug-resistance

The MTT assay of cells viability after treatment with increasing doses of anticancer drugs showed significant variations in IC50 doses between the examined cell lines. All IC50 doses are listed in Table 4. Vinblastine IC50 dose in CMT-U27 cell line was 1588 nmol, whereas after PGP and MRP1 knock-down it was only $203 \mathrm{nmol}$ and $144 \mathrm{nmol}$, respectively (Table 4, Figure 2A). In CMT-W2 cell line vinblastine IC50 was 9 434 nmol, whereas after $p g p$ and $m r p 1$ silencing it was $1264 \mathrm{nmol}$ and $1248 \mathrm{nmol}$, respectively (Table 4). We showed that after $p g p$ and mrp1 silencing IC50 was significantly lower in all the examined cell lines ranging 203-1391 nmol and 144-1248 nmol, respectively (Table 4). Significant variations were also observed in case of IC50 doses of cisplatin - the lowest IC50 dose was in CMT-U27 cell line (5 $669 \mathrm{nmol}$ ) (Figure 2B), whereas the highest was in P114 cell line (42 $944 \mathrm{nmol}$ ) 

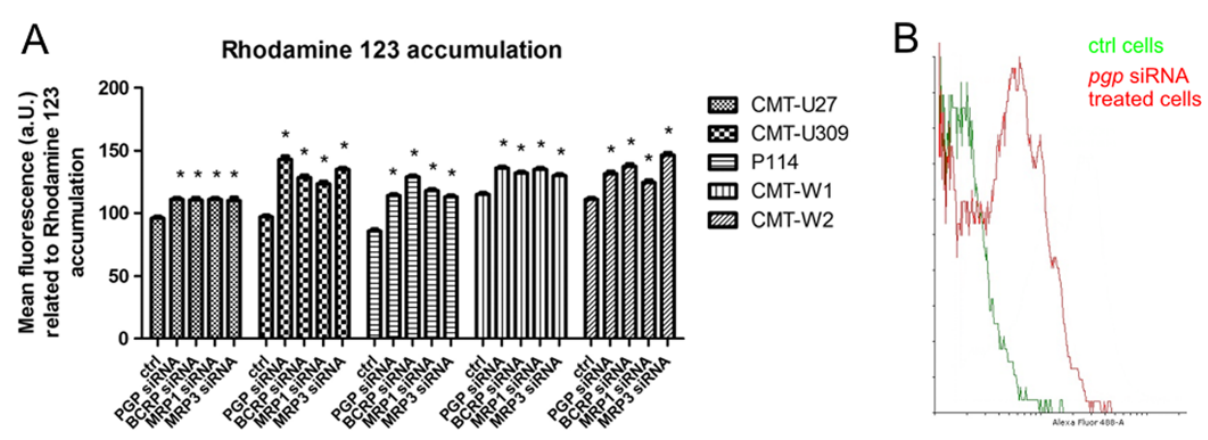

Figure 1 Activity of PGP, BCRP, MRP1 and MRP3. A.) Graph of mean fluorescence related to rhodamine-123 accumulation inside cancer cells obtained using FACS Aria II (Becton Dickinson, USA). Error bars refer to S.D. $p<0.05$ was marked as *. One-way ANOVA followed by Tukey HSD post-hoc test was applied. B.) Representative overlay histograms of rhodamine-123 accumulation in CMT-U309 canine mammary cancer cells (control, markered as green), and treated with pgp -specific siRNA (markerd as red). The overlay histograms were created using Flowing Software (Turku University, Finland), www.flowingsoftware.com.

(Table 4). Cisplatin IC50 was significantly decreased in all the examined cell lines after silencing of $p g p$, bcrp, mrp1 and mrp3 (Table 4). Regarding cyclophosphamide, we also noticed significant variations in IC50 values. It was the lowest in CMT-W1 cell line (8 $679 \mathrm{nmol})$, when on the other hand the highest in CMT-W2 cell line (25 $698 \mathrm{nmol}$ ) (Table 4). Treatment of cells with bcrpspecific siRNA decreased cyclophosphamide IC50 in all of the examined cell lines (ranging between 765 and $5705 \mathrm{nmol}$ ) (Table 4, Figure 2C).
Cancer cell treatment with anticancer drug and siRNA specific to efflux pumps increases apoptosis

The Annexin V assay was used to examine the influence of pgp, bcrp, mrp 1 and mrp3 expression knock-down on apoptosis induced by vinblastine, cisplatin and cyclophosphamide (comparing to anticancer drug given as a single agent at IC50 dose). Due to any changes in cells viability based on transfection procedure were observed in MTT assay, this kind of control has been omitted in apoptosis assay. We used similar approach in our previous study [19].

Table 4 IC50 doses of anticancer drugs in control conditions and after efflux pumps silencing

\begin{tabular}{|c|c|c|c|c|c|c|}
\hline \multirow[t]{2}{*}{ Anticancer drug } & \multirow[t]{2}{*}{ Condition } & \multicolumn{5}{|c|}{ IC50 in examined cell lines [nmol] } \\
\hline & & CMT-U27 & CMT-U309 & P114 & CMT-W1 & $\overline{C M T-W 2}$ \\
\hline \multirow[t]{5}{*}{ vinblastine } & ctrl & 1588 & 2472 & 2744 & 8072 & 9434 \\
\hline & pgp siRNA & $203^{* * *}$ & $464^{* * *}$ & $208^{* * *}$ & $1391^{* * *}$ & $1264^{* * *}$ \\
\hline & bcrp siRNA & 1497 & 2417 & 2572 & 8002 & 7346 \\
\hline & mrp1 siRNA & $144^{* * *}$ & $772^{* *}$ & $756^{* *}$ & $1747^{* * *}$ & $1248^{* * *}$ \\
\hline & mrp3 siRNA & 1330 & 2406 & 2561 & 7433 & 8482 \\
\hline \multirow[t]{5}{*}{ cisplatin } & ctrl & 5669 & 12034 & 42944 & 41288 & 9536 \\
\hline & pgp siRNA & $2203^{* *}$ & $8613^{* *}$ & $5000^{* * *}$ & $32663^{* * *}$ & $6707^{* *}$ \\
\hline & bcrp siRNA & $3300^{* *}$ & $8540^{* *}$ & $17699^{* * *}$ & $11228^{* * *}$ & $8177^{*}$ \\
\hline & mrp1 siRNA & $1339^{* *}$ & $10090^{*}$ & $20348^{* * *}$ & $29132^{* * *}$ & $5695^{* *}$ \\
\hline & mrp3 siRNA & $303^{* * *}$ & $7301^{* *}$ & $13565^{* * *}$ & $11163^{* * *}$ & $7177^{*}$ \\
\hline \multirow[t]{5}{*}{ cyclophosphamide } & ctrl & 9599 & 12542 & 10340 & 8679 & 25698 \\
\hline & pgp siRNA & 8432 & 11755 & 10242 & 7912 & 15443 \\
\hline & bcrp siRNA & $799^{* * *}$ & $2086^{* * *}$ & $5705^{* *}$ & $2463^{* *}$ & $765^{* * *}$ \\
\hline & mrp1 siRNA & 8762 & 11307 & 9955 & 7247 & 7000 \\
\hline & mrp3 siRNA & 8802 & 12278 & 10167 & 6932 & 1130 \\
\hline
\end{tabular}

IC50 doses [nmol] of anticancer drugs: vinblastine, cisplatin and cyclophosphamide in canine mammary cancer cell lines in control conditions and after silencing of efflux pumps expression. $p<0.05$ was marked as *, $p<0.01$ was marked as **, and $p<0.001$ was marked as ***. One-way ANOVA followed by Tukey HSD posthoc test was applied. 


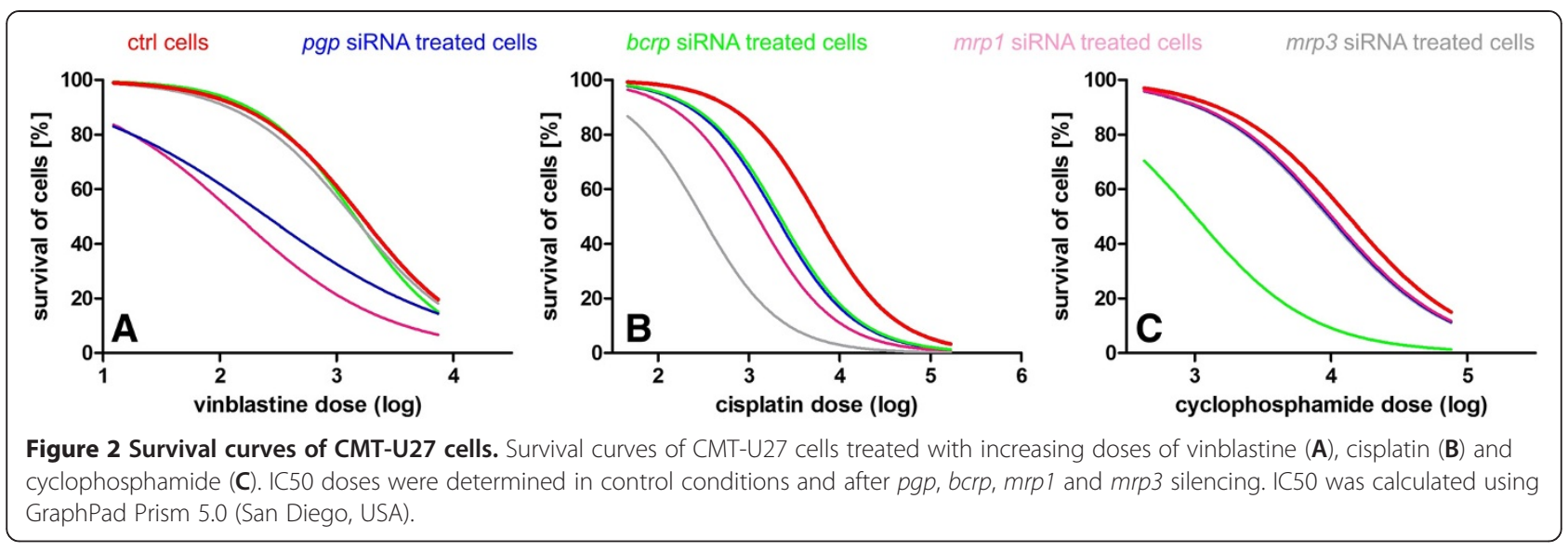

Annexin $\mathrm{V}$ analysis revealed that treatment of cells using pgp and mrpl -specific siRNA together with vinblastine (at IC50 dose) significantly increased number of apoptotic cells, compared to apoptosis caused by anticancer drug which was given as a single agent (at the IC50 dose as well) (Figure 3A). The most significant effect was pointed out in CMT-W1 cell line: $25.35 \%$ and $17.45 \%$ increase in number of apoptotic cells after $p g p$ and $m r p 1$ silencing and vinblastine treatment, respectively (Figure 3 ). Similarly, in CMT-W2 cell line $21.78 \%$ and $20.88 \%$ increase in number of apoptotic cells was shown after $p g p$ and $m r p 1$ silencing and vinblastine treatment, respectively (Figure 3 ).

In case of cisplatin treatment at IC50 dose, an increase in number of apoptotic cells was noticed in each cell line after silencing of all the efflux pumps: $p g p, b c r p, m r p 1$ and mrp3. The mostly significant result was observed in P114 cell line, where $40 \%, 41 \%, 42 \%$ and $40 \%$ increase in number of apoptotic cells $(\mathrm{p}<0.001)$ was visible, respectively (Figure 3B, D). In CMT-U309 cell line the highly significant $(p<0.001)$ effect on apoptosis was as well as in case of cisplatin treatment in cells transfected with siRNA specific to: pgp, bcrp and mrp3 (26.45\%, 26.18\% and 21.05\% increase in number of apoptotic cells, respectively), whereas significant effect $(\mathrm{p}<0.05)$ was observed in cells transfected with $m r p 1$-specific siRNA (8.25\% increase in number of apoptotic cells) (Figure 3B).

In all the examined cell lines increase in number of apoptotic cells related to cyclophosphamide treatment at IC50 dose was undoubtedly visually recognized after $b c r p$ knock-down (Figure 3C). Additionally, in CMT-W2 cell line the significant increase in number of apoptotic cells was observed due to cyclophosphamide treatment following transfection with $b c r p, m r p 1$ and $m r p 3$-specific siRNA (30\%, $12.1 \%$ and $21 \%$, respectively).

\section{Discussion}

Cancer cells retain the important mechanism of selfprotection through the activity of multiple drug resistance transporters. The multidrug resistance is frequently associated with over-expression of two or more membrane pumps that efflux anticancer drug from the cytoplasm. This protects tumor cells against the drug effects and its correlated molecular processes [8]. Expression of efflux pumps is usually higher in tumors that originate from tissues that normally show their expression and it is always higher in the tumors than in normal cells [8]. Unfortunately, the expression and role of proteins that mediate drug resistance in canine cancer cells has not yet been recognized. As increased efflux is such a significant contributor to a multidrug resistance in cancer cells, current research is aimed at blocking or inhibiting this specific mechanism. That is why the aim of our study was to assess expression of four the most important efflux pumps: PGP, BCRP, MRP1 and MRP3 in canine mammary cancer cells as well as to investigate their role in resistance to: cisplatin, cyclophosphamide and vinblastine. Because treatment of cancer cells with the use of classical inhibitors of efflux pumps often fails $[8,9]$, we used a novel approach, that is: specific RNAi to knock-down their expression.

Examination of each efflux pump expression in control conditions and after treatment with anticancer drug was also quite a novel approach. We observed significant differences in $p g p$ transcript level in control conditions and after treatment with anticancer drug. In all of the examined cell lines the $p g p$ expression increased due to vinblastine and cisplatin treatment whereas decreased due to cyclophosphamide treatment (Table 3). It ought to be outlined that these results are in accordance with IC50 doses (Table 4). In case of cisplatin treatment, expression of all four efflux pumps increased, and thus, any relationship between their transcript level and IC50 doses was very complicated to calculate (Tables 3 and 4). We also observed that cyclophosphamide treatment caused increase in bcrp expression in all of the examined cell lines. These results are in accordance with previous findings that cytotoxic drugs can initiate or increase expression of efflux pumps in cancer cells [27]. 


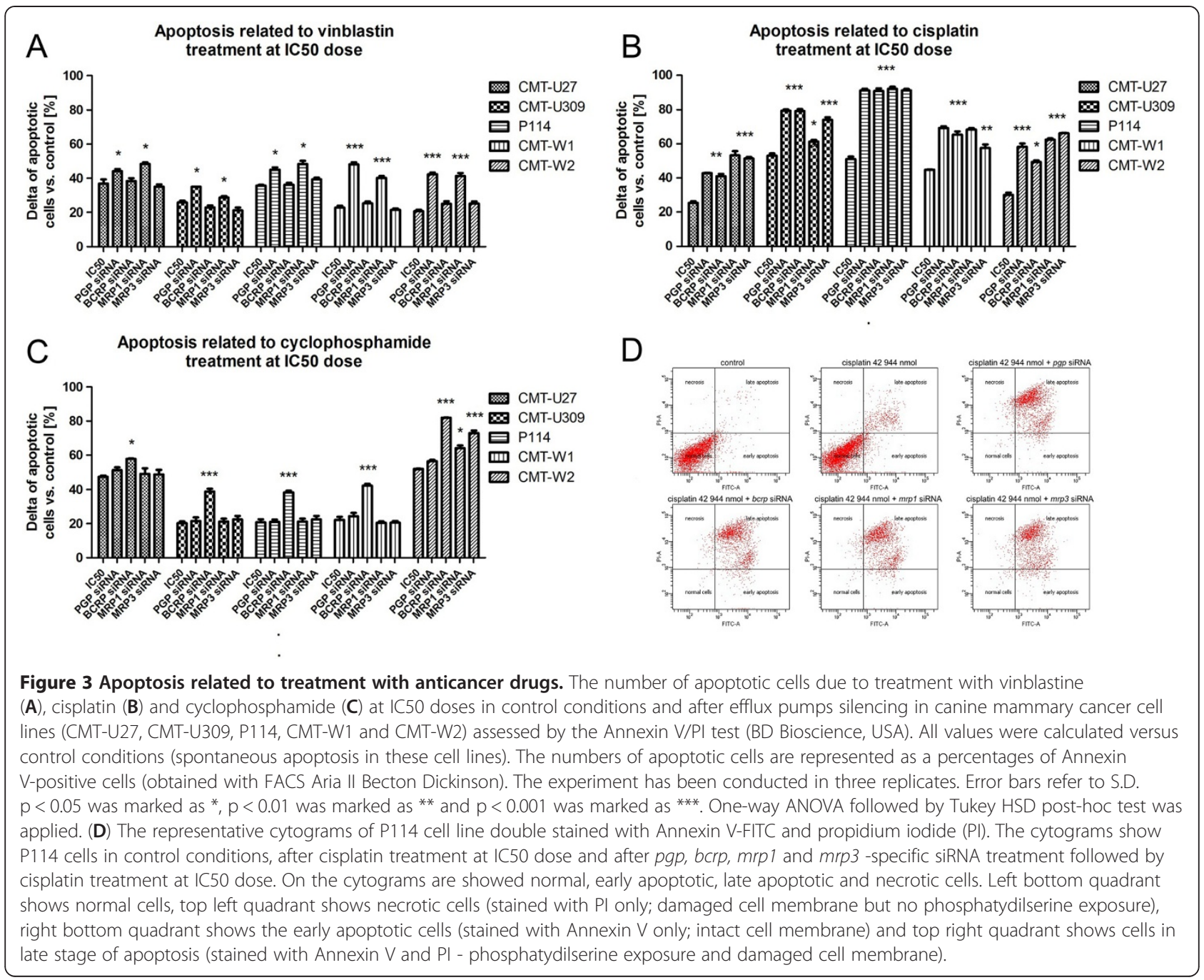

Moreover, the expression level of these efflux pumps correlates with response to anticancer therapy and patient follow up [28]. Based on our results, we have come up with the conclusion that examination of efflux pumps expression before initiation of chemotherapy could help to predict response to anticancer drugs and could be helpful in assessment of their proper doses. However, more studies in this field are required.

The RNAi gene silencing was highly successful (comparing to our previously published studies $[19,21]$ ), reaching even $100 \%$ of gene knock-down in case of bcrp in CMTW2 cell line and mrp3 in case of CMT-U309 cell line. The results of gene knock-down were confirmed by the assay with rhodamine-123 efflux. To assess whether efflux pumps silencing has an influence on cancer cell susceptibility to cytostatic drugs, the IC50 doses were determined in transfected cells (Table 4, Figure 2). Our results showed that vinblastine IC50 doses significantly decreased in all the examined cell lines after $p g p$ and mrpl silencing (Table 4). Examination of cisplatin IC50 in transfected cells pointed that it was significantly lower in all of them when compared to control cells (Table 4). In case of cyclophosphamide, bcrp siRNA treatment significantly decreased IC50 dose in all of the examined cell lines (Table 4).

Comparison of IC50 doses of anticancer drugs given in vitro and their maximum possible plasma concentrations showed that in case of vinblastine the plasma concentration was much lower than the IC50 doses [29]. It means that the examined cancer cell lines are vinblastineresistant and therefore this anticancer drug may be ineffective in canine mammary cancer treatment. However, more studies in this field should be conducted. In case of cisplatin, the IC50 doses in the three examined cell lines (CMT-U27, CMT-U309 and CMT-W2) were lower than the reachable plasma concentrations [30]. However, after bcrp, mrp1 or mrp3 knock-down, the cisplatin IC50 doses were similar or lower than the maximum possible plasma drug concentration in all of the examined cell lines. It means that knock-down of efflux pumps expression 
during chemotherapy can reverse cancer drug-resistance. The IC50 doses of cyclophosphamide in all of the examined cell lines were significantly lower than its maximum possible plasma concentration [31]. However, in all the cases given above, targeting of $\mathrm{ABC}$ efflux pumps using siRNA significantly decreased IC50 doses of anticancer drugs. These results were also confirmed by apoptosis assay (Figure 3).

In summary: knock-down of efflux pumps expression during chemotherapy could allow decreased doses of anticancer drugs thereby reducing the risk of their side effects. However, more studies in this field are required as well as large clinical trials to prove this hypothesis.

Our research is a completely new approach in the field of veterinary oncology. The results show that targeting of ABC-efflux pumps using RNAi can be promising strategy in cancer treatment. However, these in vitro studies should be confirmed using in vivo animal models.

\section{Conclusions}

The most important findings of our study are: (1) the expression level of efflux pump reflects the resistance to specific anticancer drug thus it should be assessed before the initiation of chemotherapy; (2) in canine mammary cancer PGP and MRP1 are responsible for vinblastine resistance, PGP, BCRP, MRP1 and MRP3 are cisplatin transporters whereas BCRP is a transporter of cyclophosphamide; (3) treatment of cells using siRNA targeting efflux pumps significantly increases cancer susceptibility to anticancer drug and allows to decrease the effective dose of anticancer drug.

\section{Competing interests}

The authors declare that they have no competing interests.

\section{Authors' contributions}

KP research design, experimental design, IC50 determination, FACS analyses, rhodamine-123 accumulation assay, siRNA transfections, JM apoptosis analysis, KM apoptosis analysis, TM manuscript preparation, MK research design, experimental design, FACS analyses, siRNA transfection, RT-QPCR, manuscript preparation. All authors read and approved the final manuscript.

\section{Acknowledgements}

This work was supported by grant no N N308 574940 from the Ministry of Sciences and Higher Education. The authors would like to thank Katarzyna Szyszko DVM for her help in optimization of RT-qPCR reaction conditions and Dr. Robert Meyers, M.D. for his contribution in preparation of this manuscript. The authors would like to thank Dr. Eva Hellmen (Swedish University of Life Sciences, Sweden) for her kind donation of CMT-U27 and CMT-U309 cell lines, Dr. Gerard Rutteman (Utrech University, The Netherlands) for his kind donation of P1 14 cell line and Prof. Dr. Maciej Ugorski (Wrocław University of Environmental and Life Sciences, Poland) for his kind donation of CMT-W1 and CMT-W2 cell lines.

\section{Author details}

'Department of Physiological Sciences, Faculty of Veterinary Medicine, Warsaw University of Life Sciences - WULS, Nowoursynowska 159, 02-776, Warsaw, Poland. ${ }^{2}$ Department of Large Animal Diseases with Clinic, Faculty of Veterinary Medicine, Warsaw University of Life Sciences - WULS, Nowoursynowska 100, 02-797, Warsaw, Poland. ${ }^{3}$ Department of Animal Environment Biology, Faculty of Animal Sciences, Warsaw University of Life Sciences - WULS, Ciszewskiego 8, 02-786, Warsaw, Poland.
Received: 12 January 2013 Accepted: 13 June 2013

Published: 17 June 2013

\section{References}

1. MacEwen EG: Spontaneous tumors in dogs and cats: models for the study of cancer biology and treatment. Cancer Metastasis Rev 1990, 9:125-136.

2. Midsorp W: Tumors of the mammary gland. In Tumors in domestic animals. Edited by Meuten DJ. Ames: Iowa State Press; 2002:575-606.

3. Misdorp W: Progestagens and mammary tumours in dogs and cats. Acta Endocrinol 1991, 125(Suppl. 1):27-31.

4. Stratmann N, Failing K, Richter A, Wehrend A: Mammary tumor recurrence in bitches after regional mastectomy. Vet Surg 2008, 37:82-86.

5. Simon D, Schoenrock D, Baumgartner W, Nolte I: Postoperative adjuvant treatment of invasive malignant mammary gland tumors in dogs with doxorubicin and docetaxel. J Vet Intern Med 2006, 20:1184-1190.

6. Marconato L, Lorenzo RM, Abramo F, Ratto A, Zini E: Adjuvant gemcitabine after removal of aggressive malignant mammary tumours in dogs. Vet Comp Oncol 2008, 6:90-101

7. Karayannopoulou M, Kaldrymidou E, Constantinidis TC, Dessiris A: Adjuvant post-operative chemotherapy in bitches with mammary cancer. Transbound Emerg Dis 2001, 48:85-96.

8. Król M, Pawłowski KM, Majchrzak K, Szyszko K, Motyl T: Why chemotherapy can fail? Pol J Vet Sci 2010, 12:399-406.

9. Szyszko K, Pawłowski KM, Motyl T, Król M: Chemoresistance in cancer: not just a humans problem. Med Wet 2011, 67(7):453-457.

10. Honscha KU, Schirmer A, Reischauer A, Schoon HA, Einspanier A, Gabel G: Expression of $A B C$-transport proteins in canine mammary cancer: consequences for chemotherapy. Reprod Domest Anim 2009, 44:218-223.

11. Roth BJ, Sledge GW, Williams SD, Meyer SC, Ansari R, Fisher WB: Methotrexate, Vinblastine, Doxorubicin, and Cisplatin in metastatic breast cancer. Cancer 1991, 68:248-252.

12. Briest S, Stearns V: Chemotherapeutic strategies for advanced breast cancer. Oncology 2007, 21(11):1325-1335.

13. Ospovat I, Siegelmann-Danieli N, Grenader T, Hubert A, Hamburger T, Peretz $\mathrm{T}$ : Mitomycin $\mathrm{C}$ and vinblastine: an active regimen in previously treated breast cancer patients. Tumori 2009, 95(6):683-686.

14. Whitehead KA, Langer $R$, Anderson DG: Knocking down barriers: advances in siRNA delivery. Nat Rev Drug Discovery 2009, 8:129-138.

15. Król M, Pawłowski KM, Majchrzak K, Gajewska M, Majewska A, Motyl T: Global gene expression profiles of canine macrophages and canine mammary cancer cells grown as a co-culture in vitro. BMC Vet Res 2012, 8:16.

16. Król M, Pawłowski KM, Skierski J, Rao NAS, Hellmen E, Mol JA, Motyl T: Transcriptomic profile of two canine mammary cancer cell lines with different proliferative and anti-apoptotic potential. J Physiol Pharmacol 2009, 60:95-106.

17. Król M, Pawłowski KM, Skierski J, Turowski P, Majewska A, Polańska J, Ugorski M, Morty RE, Motyl T: Transcriptomic "portraits" of canine mammary cancer cell lines with various phenotype. J App/ Genet 2010, 51:169-183.

18. Król M, Polańska J, Pawłowski KM, Skierski J, Majewska A, Ugorski M, Motyl T: Molecular signature of cell lines isolated from mammary adenocarcinoma metastases to lungs. J Appl Genet 2010, 51:37-50.

19. Pawłowski KM, Popielarz D, Szyszko K, Motyl T, Król M: Growth Hormone Receptor RNA interference decreases proliferation and enhances apoptosis in canine mammary carcinoma cell line CMT-U27. Vet Comp Oncol 2012, 10(1):2-15.

20. Majchrzak K, Pawlowski KM, Orzechowska EJ, Dolka I, Mucha J, Motyl T, Król $\mathrm{M}$ : A role of ghrelin in canine mam mary carcinoma cells proliferation, apoptosis and migration. BMC Vet Res 2012, 8:170.

21. Król M, Majchrzak K, Mucha J, Homa A, Bulkowska M, Jakubowska A, Karwicka M, Pawłowski KM, Motyl T: CSF-1R as an inhibitor of apoptosis and promoter of proliferation, migration and invasion of canine mammary cancer cells. BMC Vet Res 2013, 9:65.

22. Brinkhof B, Spee B, Rothuizen J, Penning LC: Development and evaluation of canine reference genes for accurate quantification of gene expression. Anal Biochem 2006, 356:36-43.

23. Etschmann B, Wilcken B, Stoevesand K, von der Schulenburg A, Sterner-Kock $A$ : Selection of reference genes for quantitative real-time PCR analysis in canine mammary tumors using the GeNorm algorithm. Vet Pathol 2006, 43:934-942.

24. Schmittgen TD, Livak KJ: Analyzing real-time PCR data by the comparative Ct method. Nature Prot 2008, 3:1101-1108. 
25. Palmeira A, Vasconcelos MH, Paiva A, Fernandes MX, Pinto M, Sousa E: Dual inhibitors of P-glycoprotein and tumor cell growth: (Re)discovering thioxanthones. Biochem Pharmacol 2012, 83:57-68.

26. Nalbantsoy A, Karabay-Yavasoglu NU, Sayim F, Deliloglu-Gurhan I, Gocmen B, Arikan H, Yildiz MZ: Determination of in vivo toxicity and in vitro cytotoxicity of venom from the Cypriot blunt-nosed viper Macrovipera lebetina lebetina and antivenom production. JVATTD 2012, 18:208-216.

27. Grube S, Langguth P: Modulation of drug-carrier activity by excipients In Drug delivery research advances. Edited by Mashkevich BO. New York: Nova Science Publishers, Inc; 2007:81-104. ISBN 978-1-60021-732-6.

28. Sun J, He ZG, Cheng G, Wang SJ, Hao XH, Zou MJ: Multidrug resistance Pglycoprotein: crucial significance in drug disposition and interaction. Med Sci Monit 2004, 10:RA5-RA14.

29. Creasey W, Scott Al, Wei CC, Kutcher J, Schwartz A, Marsh JC:

Pharmacological studies with Vinblastine in the dog. Cancer Res 1975, 35:1116-1120

30. Schroeder A, Honen R, Turjeman K, Gabizon A, Kost J, Barenholz Y: Ultrasound triggered release of cisplatin from liposomes in murine tumors. J Control Release 2009, 137(1):63-68.

31. Struck RF, Alberts DS, Horne K, Phillips JG, Peng YM, Roe DJ: Plasma pharmacokinetics of cyclophosphamide and its cytotoxic metabolites after intravenous versus oral administration in a randomized, crossover trial. Cancer Res 1987, 47:2723-2726.

doi:10.1186/1746-6148-9-119

Cite this article as: Pawłowski et al: Expression and role of PGP, BCRP, MRP1 and MRP3 in multidrug resistance of canine mammary cancer cells. BMC Veterinary Research 2013 9:119.

\section{Submit your next manuscript to BioMed Central and take full advantage of:}

- Convenient online submission

- Thorough peer review

- No space constraints or color figure charges

- Immediate publication on acceptance

- Inclusion in PubMed, CAS, Scopus and Google Scholar

- Research which is freely available for redistribution 